\title{
Fractional integral operator on modulation and Wiener amalgam spaces
}

\author{
Meifang Cheng* and Wenyu Tao
}

${ }^{*}$ Correspondence:

cmf78529@mail.ahnu.edu.cn

Department of Mathematics and Computer Science, Anhui Normal

University, Wuhu, 241000, P.R. China

\begin{abstract}
The purpose of this paper is to investigate the mapping properties of the fractional integral operators on weighted modulation spaces. Based on this result, we also study the boundedness of the bilinear fractional integral operators on product Wiener amalgam spaces. Our results show that, besides modulation spaces, Wiener amalgam spaces are good substitutions for Lebesgue spaces.
\end{abstract}

MSC: Primary 42B20; 42B25

Keywords: fractional integral operator; weighted modulation space; bilinear fractional integral operator; Wiener amalgam space

\section{Introduction}

Time-frequency analysis is a modern branch of harmonic analysis. It has many applications in signal analysis and wireless communication (see $[1,2])$. Time-frequency analysis is a form of local Fourier analysis that treats time and frequency simultaneously. Inspired by this idea, modulation and Wiener amalgam spaces have been introduced and used to measure the time-frequency concentration of a function or a tempered distribution (see [3-7]). During the last ten years, these two function spaces have not only become useful function spaces for time-frequency analysis, they have also been employed to study boundedness properties of pseudo-differential operators, Fourier multipliers, Fourier integral operators, and well-posedness of solutions to PDEs. For more details of the applications of these two function spaces, the reader is referred to [8-19] and the references therein.

In this paper, we are mainly concerned with the mapping properties of the fractional integral operator on weighted modulation spaces. Using this result, we also prove the boundedness of the bilinear fractional integral operator on product Wiener amalgam spaces. From our results, we will see that, besides modulation spaces, Wiener amalgam spaces are good substitutions for Lebesgue spaces.

The fractional integral operator $I_{\alpha}$ is defined by

$$
I_{\alpha} f(x)=\frac{1}{\gamma(\alpha)} \int_{\mathbb{R}^{n}} \frac{f(y)}{|x-y|^{n-\alpha}} d y, \quad \gamma(\alpha)=\frac{\pi^{\frac{n}{2}} \Gamma\left(\frac{\alpha}{2}\right) 2^{\alpha}}{\Gamma\left(\frac{n-\alpha}{2}\right)},
$$

(c) 2015 Cheng and Tao. This article is distributed under the terms of the Creative Commons Attribution 4.0 International License (http://creativecommons.org/licenses/by/4.0/), which permits unrestricted use, distribution, and reproduction in any medium, provided you give appropriate credit to the original author(s) and the source, provide a link to the Creative Commons license, and indicate if changes were made. 
where $0<\alpha<n$. The well-known Hardy-Littlewood-Sobolev theorem says that $I_{\alpha}$ is bounded from $L^{p}\left(\mathbb{R}^{n}\right)$ to $L^{q}\left(\mathbb{R}^{n}\right)$ for $1<p<q<\infty$ and $\frac{1}{q}=\frac{1}{p}-\frac{\alpha}{n}$. This theorem plays important roles in partial differential equations.

In recent years, many authors were interested in the mapping properties of the fractional integral operator on modulation spaces. For example, in [20], Tomita regarded the operator $I_{\alpha}$ as a special case of pseudo-differential operator and proved the following results. Set $0<\alpha<n, 0<\varepsilon<1-\frac{\alpha}{n}$, and $0<\rho<\frac{\alpha}{n}$. If $1<p_{1}, p_{2}, q_{1}, q_{2}<\infty$ satisfy

$$
\frac{1}{q_{1}}=\frac{1}{p_{1}}-\frac{\alpha}{n}-\varepsilon \text { and } \quad \frac{1}{q_{2}}=\frac{1}{p_{2}}+\frac{\alpha}{n}-\rho,
$$

then there exists a constant $C_{\alpha, \varepsilon, \rho}>0$, such that

$$
\left\|I_{\alpha} f\right\|_{M^{q_{1}, q_{2}\left(\mathbb{R}^{n}\right)} \leq C_{\alpha, \varepsilon, \rho}}\|f\|_{M^{p_{1}, p_{2}\left(\mathbb{R}^{n}\right)}}
$$

for all $f \in \mathcal{S}\left(\mathbb{R}^{n}\right)$.

Subsequently, Sugimoto and Tomita improved the results in [20] and obtained necessary and sufficient conditions for the boundedness of the operator $I_{\alpha}$ (see [21]). Using the definitions of the discrete form for modulation spaces, which will be given by Definition 2.4 in the next section, they proved that $I_{\alpha}$ is bounded from $M^{p_{1}, q_{1}}\left(\mathbb{R}^{n}\right)$ to $M^{p_{2}, q_{2}}\left(\mathbb{R}^{n}\right)$ if and only if

$$
\frac{1}{p_{2}} \leq \frac{1}{p_{1}}-\frac{\alpha}{n}, \quad \frac{1}{q_{2}}<\frac{1}{q_{1}}+\frac{\alpha}{n}
$$

where $0<\alpha<n$ and $1<p_{1}, p_{2}, q_{1}, q_{2}<\infty$.

Recently, in [22], by using the norm of Hardy spaces $H^{p}$, Chen and Zhong introduced the modulation Hardy spaces $M^{p, q}$ for $0<p \leq 1$ and $0<q \leq \infty$. They proved that if $\frac{n p}{n-\alpha p} \leq$ $r \leq \infty$, then $I_{\alpha}$ is bounded from $M_{-\alpha}^{p, q}$ to $M^{r, q}$ for $0<p \leq 1$ and $0<q \leq \infty$. Moreover, for $1<p<\infty$ and $0<q_{1}, q_{2} \leq \infty$, they obtained $I_{\alpha}$ is bounded from $M^{p, q_{1}}$ to $M^{\infty, q_{2}}$ if and only if

$$
\frac{1}{p}>\frac{\alpha}{n}, \quad \frac{1}{q_{2}}<\frac{1}{q_{1}}+\frac{\alpha}{n} .
$$

In their proofs, Chen and Zhong also used the definition of the discrete form for modulation spaces.

Inspired by Sugimoto and Tomita, using the definition of integral form for modulation spaces, which will be given by Definition 2.3 in Section 2, we prove the following result.

Theorem 1.1 For $0<\alpha<n$ and $s_{1}, s_{2} \in \mathbb{R}$, let $1<p_{1}, p_{2}<\infty$ and $0<q_{1}, q_{2} \leq \infty$. If

$$
\frac{1}{p_{2}} \leq \frac{1}{p_{1}}-\frac{\alpha}{n}, \quad \frac{1}{q_{2}}<\frac{1}{q_{1}}+\frac{\alpha}{n} \quad \text { and } \quad s_{2}<s_{1}+n\left(\frac{1}{q_{1}}-\frac{1}{q_{2}}\right)
$$

then the fractional integral operator $I_{\alpha}$ is bounded from $M_{s_{1}}^{p_{1}, q_{1}}\left(\mathbb{R}^{n}\right)$ to $M_{s_{2}}^{p_{2}, q_{2}}\left(\mathbb{R}^{n}\right)$. 
Another purpose of this paper is to study the mapping properties of the bilinear fractional integral operator $B_{\alpha}$, which is defined by

$$
B_{\alpha}(f, g)(x)=\int_{\mathbb{R}^{n}} \frac{f(x-y) g(x+y)}{|y|^{n-\alpha}} d y,
$$

on modulation spaces. It was showed in [22] that if $1<p<\frac{n}{n-\alpha}$ and $0<q \leq \infty$, then

$$
\left\|\widehat{B_{\alpha}(f, g)}\right\|_{M^{\infty, \infty}} \leq C\|f\|_{M^{1,1}}\left\|\mathcal{F}^{-1}(g)\right\|_{M^{p, q}} .
$$

In particular, for $p \leq q$,

$$
\left\|B_{\alpha}(f, g)\right\|_{M^{\infty, \infty}} \leq C\|f\|_{M^{1,1}}\|g\|_{M^{q, p}} .
$$

Inspired by Chen and Zhong, in this paper, we investigate the boundedness of the bilinear fractional integral operator $B_{\alpha}$ on Wiener amalgam spaces. Our result is as follows.

Theorem 1.2 For $0<\alpha<n, 1<p_{0}, p_{1}<\infty$, and $1 \leq q_{0}, q_{1}, p_{2}, q_{2}<\infty$, let $p_{0}^{\prime}$ and $q_{0}^{\prime}$ denote the conjugate index of $p_{0}$ and $q_{0}$, respectively. Suppose $q_{0}^{\prime} \geq p_{0}^{\prime}, \frac{1}{p_{0}} \leq \frac{1}{p_{1}}-\frac{n-\alpha}{n}, \frac{1}{q_{0}}<\frac{1}{q_{1}}+$ $\frac{n-\alpha}{n}$, and $\frac{1}{q_{0}^{\prime}}+\frac{1}{p_{0}^{\prime}}=\frac{1}{p_{2}}+\frac{1}{q_{2}}$, then the operator $B_{\alpha}$ maps $W\left(\mathcal{F} L^{p_{1}}, L^{q_{1}}\right) \times W\left(\mathcal{F} L^{p_{2}}, L^{p_{0}^{\prime}}\right)$ to $W\left(\mathcal{F} L^{q_{2}^{\prime}}, L^{q_{0}}\right)$.

In what follows, we always denote $C$ to be a positive constant that may be different at each place, but is independent of the essential variables.

This paper is organized as follows. In Section 2, we give the definitions and basic properties of modulation and Wiener amalgam spaces. Section 3 is devoted to the proofs of our main results.

\section{Basic definitions and important lemmas}

The following notations will be used throughout this paper. Let $\mathcal{S}\left(\mathbb{R}^{n}\right)$ be the Schwartz space of all complex-valued rapidly decreasing infinitely differentiable functions on $\mathbb{R}^{n}$ and $\mathcal{S}^{\prime}\left(\mathbb{R}^{n}\right)$ be the topological dual of $\mathcal{S}\left(\mathbb{R}^{n}\right)$. For a function $f$ in $\mathcal{S}\left(\mathbb{R}^{n}\right)$, its Fourier transform is defined by $\hat{f}(\omega)=\int f(t) e^{-2 \pi i \omega \cdot t} d t$, and its inverse Fourier transform is $\breve{f}(t)=\hat{f}(-t)$. The translation and the modulation operators are defined by

$$
T_{x} f(t)=f(t-x) \quad \text { and } \quad M_{\omega} f(t)=e^{2 \pi i \omega \cdot t} f(t)
$$

for every $x, \omega \in \mathbb{R}^{n}$. For $s \in \mathbb{R}$ and $x \in \mathbb{R}^{n}$, the weight function $\langle x\rangle^{s}=\left(1+|x|^{2}\right)^{\frac{s}{2}}$.

Definition 2.1 Let $g$ be a non-zero Schwartz function and $1 \leq p, q \leq \infty$ and $s \in \mathbb{R}$, the weighted modulation space $M_{s}^{p, q}\left(\mathbb{R}^{n}\right)$ is defined as the closure of the Schwartz class with respect to the norm

$$
\|f\|_{M_{s}^{p, q}}=\left(\int_{\mathbb{R}^{n}}\left(\int_{\mathbb{R}^{n}}\left|V_{g} f(x, w)\right|^{p} d x\right)^{\frac{q}{p}}\langle w\rangle^{s q} d w\right)^{\frac{1}{q}},
$$


with obvious modifications for $p$ or $q=\infty$, where $V_{g} f(x, w)$ is the so-called short time Fourier transform (STFT), which is defined by

$$
V_{g} f(x, w)=\left\langle f, M_{\xi} T_{x} g\right\rangle=\int e^{-2 \pi i w \cdot y} f(y) \overline{g(y-x)} d y,
$$

i.e. the Fourier transform $\mathcal{F}$ applied to $f \overline{T_{x} g}$.

Recently, the above definition has been generalized by Kobayashi in [23] to the case $0<p, q \leq \infty$. In his definition, the function $g$ is restricted to the space $\Phi^{\delta}\left(\mathbb{R}^{n}\right)$, which is defined as follows.

Definition 2.2 For $\delta>0$, we define $\Phi^{\delta}\left(\mathbb{R}^{n}\right)$ to be the space of all $g \in \mathcal{S}\left(\mathbb{R}^{n}\right)$ satisfying

$$
\operatorname{supp} \hat{g} \subset\{\xi:|\xi| \leq 1\} \quad \text { and } \quad \sum_{k \in \mathbb{Z}^{n}} \hat{g}(\xi-\delta k)=1
$$

We may choose a sufficiently small $\delta$, such that the function space $\Phi^{\delta}\left(\mathbb{R}^{n}\right)$ is not empty.

Definition 2.3 Given a $g \in \Phi^{\delta}\left(\mathbb{R}^{n}\right)$, and $0<p, q \leq \infty, s \in \mathbb{R}$, we define the modulation space $M_{s}^{p, q}\left(\mathbb{R}^{n}\right)$ to be the space of all tempered distributions $f \in \mathcal{S}^{\prime}\left(\mathbb{R}^{n}\right)$ such that the quasinorm

$$
\|f\|_{M_{s}^{p, q}}=\left(\int_{\mathbb{R}^{n}}\left(\int_{\mathbb{R}^{n}}\left|f *\left(M_{w} g\right)(x)\right|^{p} d x\right)^{\frac{q}{p}}\langle w\rangle^{s q} d w\right)^{\frac{1}{q}}
$$

is finite, with obvious modifications for $p$ or $q=\infty$.

For a more general definition, involving different kinds of weight functions, both in the time and the frequency variables we refer the reader to [24]. Definitions 2.1 and 2.3 are the integral form. We also have the definition of discrete form for modulation spaces, which is very useful in studying unimodular Fourier multipliers.

Definition 2.4 Let $1 \leq p, q \leq \infty, s \in \mathbb{R}$, and $\phi \in \mathcal{S}\left(\mathbb{R}^{n}\right)$ be such that

$$
\operatorname{supp} \phi \subset[-1,1]^{n}, \quad \sum_{k \in \mathbb{Z}^{n}} \phi(\xi-k)=1
$$

for all $\xi \in \mathbb{R}^{n}$. Denote $\phi_{k}(\xi)=\phi(\xi-k)$ and let $\phi_{k}(D)$ be the Fourier multiplier operator given by $\widehat{\phi_{k}(D) f}(\xi)=\phi_{k}(\xi) \hat{f}(\xi)$. Then the weighted modulation space $M_{s}^{p, q}\left(\mathbb{R}^{n}\right)$ consists of all $f \in \mathcal{S}^{\prime}\left(\mathbb{R}^{n}\right)$ such that

$$
\|f\|_{M_{s}^{p, q}}=\left(\sum_{k \in \mathbb{Z}^{n}}(1+|k|)^{s q}\left\|\phi_{k}(D) f\right\|_{p}^{q}\right)^{\frac{1}{q}}<\infty
$$

with obvious modifications for $p$ or $q=\infty$.

There is yet another definition of modulation spaces, which is given by Gabor frames and plays a key role in studying simultaneously the local time and global frequency behavior of functions (see [24]). 
The following basic properties of weighted modulation spaces, which play important roles in this article, can be found in [23, 25].

Lemma 2.5 Let $0<p, q \leq \infty$ and $g \in \Phi^{\delta}\left(\mathbb{R}^{n}\right)$. Then

(1) Different test functions $g_{1}, g_{2} \in \Phi^{\delta}\left(\mathbb{R}^{n}\right)$ define the same spaces and equivalent quasi-norms on $M_{s}^{p, q}\left(\mathbb{R}^{n}\right)$.

(2) Let $0<p_{0} \leq p_{1} \leq \infty, 0<q_{0} \leq q_{1} \leq \infty$ and $s \in \mathbb{R}$, then

$$
M_{s}^{p_{0}, q_{0}}\left(\mathbb{R}^{n}\right) \hookrightarrow M_{s}^{p_{1}, q_{1}}\left(\mathbb{R}^{n}\right)
$$

(3) If $0<p, q<\infty$, then $\mathcal{S}\left(\mathbb{R}^{n}\right)$ is dense in $M_{s}^{p, q}\left(\mathbb{R}^{n}\right)$.

(4) For $1 \leq p, q<\infty$ and $s \in \mathbb{R},\left(M_{s}^{p, q}\right)^{\prime}=M_{-s}^{p^{\prime}, q^{\prime}}$.

To prove our main results, we also need the definition of the Wiener amalgam space $W\left(\mathcal{F} L^{p}, L^{q}\right)\left(\mathbb{R}^{n}\right)$.

For $1 \leq p \leq \infty$, let $\mathcal{F} L^{p}\left(\mathbb{R}^{n}\right)$ be the space of tempered distributions with their Fourier transforms in $L^{p}\left(\mathbb{R}^{n}\right)$, that is,

$$
\mathcal{F} L^{p}\left(\mathbb{R}^{n}\right)=\left\{f \in \mathcal{S}^{\prime}\left(\mathbb{R}^{n}\right) \mid \hat{f} \in L^{p}\left(\mathbb{R}^{n}\right)\right\}
$$

with norm $\|f\|_{\mathcal{F} L^{p}}=\|\hat{f}\|_{L^{p}\left(\mathbb{R}^{n}\right)}$.

Definition 2.6 For $1 \leq p, q<\infty$, a tempered distribution $f$ is in the Wiener amalgam spaces $W\left(\mathcal{F} L^{p}, L^{q}\right)\left(\mathbb{R}^{n}\right)$, if $f$ is locally in $\mathcal{F} L^{p}\left(\mathbb{R}^{n}\right)$, that is, for every non-zero $g \in C_{0}^{\infty}\left(\mathbb{R}^{n}\right)$, $\mathcal{F}\left(f T_{x} g\right) \in L^{p}\left(\mathbb{R}^{n}\right)$, and

$$
\|f\|_{W\left(\mathcal{F} L^{p}, L^{q}\right)}=\left(\int_{\mathbb{R}^{n}}\left(\int_{\mathbb{R}^{n}}\left|\mathcal{F}\left(f T_{x} g\right)(y)\right|^{p} d y\right)^{\frac{q}{p}} d x\right)^{\frac{1}{q}}
$$

is finite, with obvious modifications for $p$ or $q=\infty$. This definition is independent of the choice of the test function $g \in C_{0}^{\infty}\left(\mathbb{R}^{n}\right)$.

Both the modulation spaces and the Wiener amalgam spaces are mixed-norm function spaces. The following lemma gives the relationship between them.

Lemma 2.7 Let $\mathcal{F}$ be the Fourier transform and $1 \leq p, q \leq \infty$, then $M^{p, q}\left(\mathbb{R}^{n}\right)=\mathcal{F} W\left(\mathcal{F} L^{p}\right.$, $\left.L^{q}\right)\left(\mathbb{R}^{n}\right)$.

Proof Choose $g \in \mathcal{S}\left(\mathbb{R}^{n}\right)$ such that $g \neq 0$ and $f \in \mathcal{S}^{\prime}\left(\mathbb{R}^{n}\right)$. For every $x, \xi \in \mathbb{R}^{n}$, the definition of the short time Fourier transform implies that

$$
V_{g} f(x, \xi)=e^{-2 \pi i x \cdot \xi} V_{\hat{g}} \hat{f}(\xi,-x)
$$

and

$$
V_{\hat{g}} \hat{f}(\xi,-x)=\mathcal{F}\left(\hat{f} \overline{T_{\xi} \hat{g}}\right)(-x)
$$


Therefore, Definitions 2.1 and 2.6 yield

$$
\begin{aligned}
\|f\|_{M^{p}, q} & =\left(\int_{\mathbb{R}^{n}}\left(\int_{\mathbb{R}^{n}}\left|V_{g} f(x, \xi)\right|^{p} d x\right)^{\frac{q}{p}} d \xi\right)^{\frac{1}{q}} \\
& =\left(\int_{\mathbb{R}^{n}}\left(\int_{\mathbb{R}^{n}}\left|V_{\hat{g}}(\hat{f})(\xi,-x)\right|^{p} d x\right)^{\frac{q}{p}} d \xi\right)^{\frac{1}{q}} \\
& =\left(\int_{\mathbb{R}^{n}}\left(\int_{\mathbb{R}^{n}}\left|\mathcal{F}\left(\hat{f} \overline{T_{\xi} \hat{g}}\right)(-x)\right|^{p} d x\right)^{\frac{q}{p}} d \xi\right)^{\frac{1}{q}} \\
& =\|\hat{f}\|_{W\left(\mathcal{F} L^{p}, L^{q}\right) .}
\end{aligned}
$$

The proof of Lemma 2.7 is completed.

\section{Proof of the main results}

In this section, we are going to prove our main results. First, we show the proof of Theorem 1.1.

Set $K_{\alpha}(x)=\frac{1}{\gamma(\alpha)} \frac{1}{|x|^{n-\alpha}}$, where $\gamma(\alpha)=\frac{\pi^{\frac{n}{2}} \Gamma\left(\frac{\alpha}{2}\right) 2^{\alpha}}{\Gamma\left(\frac{n-\alpha}{2}\right)}$. The fractional integral operator $I_{\alpha}$, which is defined by $I_{\alpha}(f)(x)=\left(K_{\alpha} * f\right)(x)$, may be realized on the transform side as a Fourier multiplier

$$
\widehat{I_{\alpha}(f)}(\xi)=m_{\alpha}(\xi) \hat{f}(\xi)
$$

where $m_{\alpha}(\xi)=\widehat{K_{\alpha}}(\xi)$.

Proof We consider the following three cases to obtain Theorem 1.1.

Case 1: $\frac{1}{p_{2}}=\frac{1}{p_{1}}-\frac{\alpha}{n}$ and $q_{1}>q_{2}$.

Let $g, \chi \in \Phi^{\delta}\left(\mathbb{R}^{n}\right)$ satisfy the condition $g=\chi * \chi$. Then $M_{\xi} g=M_{\xi} \chi * M_{\xi} \chi$. Young's inequalities and the Hardy-Littlewood-Sobolev theorem give

$$
\begin{aligned}
\left\|I_{\alpha}(f)\right\|_{M_{s_{2}}^{p_{2}, q_{2}}} & =\left(\int_{\mathbb{R}^{n}}\left\|I_{\alpha}(f) * M_{\xi} g\right\|_{L^{p_{2}}}^{q_{2}}\langle\xi\rangle^{q_{2} s_{2}} d \xi\right)^{\frac{1}{q_{2}}} \\
& \leq\left(\int_{\mathbb{R}^{n}}\left\|\left|K_{\alpha} * f * M_{\xi} \chi\right| *\left|M_{\xi} \chi\right|\right\|_{L^{p_{2}}}^{q_{2}}\langle\xi\rangle^{q_{2} s_{2}} d \xi\right)^{\frac{1}{q_{2}}} \\
& \leq C\|\chi\|_{L^{1}}\left(\int_{\mathbb{R}^{n}}\left\|f * M_{\xi} \chi\right\|_{L^{p_{1}}}^{q_{2}}\langle\xi\rangle^{q_{2} s_{2}} d \xi\right)^{\frac{1}{q_{2}}} .
\end{aligned}
$$

Using the Hölder inequalities for the exponent $\frac{q_{1}}{q_{2}}$ and $\left(\frac{q_{1}}{q_{2}}\right)^{\prime}$, we get

$$
\begin{aligned}
& \left\|I_{\alpha} f\right\|_{M_{s_{2}}^{p_{2}, q_{2}}} \leq C\|\chi\|_{L^{1}}\left(\int_{\mathbb{R}^{n}}\left\|f * M_{\xi} \chi\right\|_{L^{p_{1}}}^{q_{2}}\langle\xi\rangle^{q_{2} s_{1}}\langle\xi\rangle^{q_{2}\left(s_{2}-s_{1}\right)} d \xi\right)^{\frac{1}{q_{2}}} \\
& \leq C\|\chi\|_{L^{1}}\left(\int_{\mathbb{R}^{n}}\left(\left\|f * M_{\xi} \chi\right\|_{L^{p_{1}}}\langle\xi\rangle^{s_{1}}\right)^{q_{2} \frac{q_{1}}{q_{2}}} d \xi\right)^{\frac{q_{2}}{q_{1}} \frac{1}{q_{2}}} \\
& \times\left(\int_{\mathbb{R}^{n}}\langle\xi\rangle^{\left(s_{2}-s_{1}\right) \frac{q_{1} q_{2}}{q_{1}-q_{2}}} d \xi\right)^{\frac{1}{q_{2}} \frac{q_{1}-q_{2}}{q_{1}}} .
\end{aligned}
$$


Denote

$$
J=\left(\int_{\mathbb{R}^{n}}\langle\xi\rangle^{\left(s_{2}-s_{1}\right) \frac{q_{1} q_{2}}{q_{1}-q_{2}}} d \xi\right)^{\frac{1}{q_{2}} \frac{q_{1}-q_{2}}{q_{1}}} .
$$

Since $s_{2}<s_{1}+n\left(\frac{1}{q_{1}}-\frac{1}{q_{2}}\right)$, an easy computation shows that

$$
\begin{aligned}
J & =\left[\left(\int_{|\xi| \leq 1}+\int_{|\xi|>1}\right)\langle\xi\rangle^{\left(s_{2}-s_{1}\right) \frac{q_{1} q_{2}}{q_{1}-q_{2}}} d \xi\right]^{\frac{q_{1}-q_{2}}{q_{1} q_{2}}} \\
& \leq C_{n}+\left(\int_{S^{n-1}} \int_{1}^{\infty} \rho^{\left(s_{2}-S_{1}\right) \frac{q_{1} q_{2}}{q_{1}-q_{2}}} \rho^{n-1} d \rho d \xi^{\prime}\right)^{\frac{q_{1}-q_{2}}{q_{1} q_{2}}} \\
& \leq C_{n}<\infty
\end{aligned}
$$

Therefore,

$$
\left\|I_{\alpha} f\right\|_{M_{s_{2}}^{p_{2}, q_{2}}} \leq C\|f\|_{M_{s_{1}}^{p_{1}, q_{1}}}
$$

Case 2: $\frac{1}{p_{2}}=\frac{1}{p_{1}}-\frac{\alpha}{n}$ and $q_{1} \leq q_{2}$. Since $0<\frac{\alpha}{n}<1$, we can choose $0<\tilde{q}_{2} \leq \infty$ such that $q_{1}>\widetilde{q_{2}}$ and $\frac{1}{\widetilde{q_{2}}}<\frac{1}{q_{1}}+\frac{\alpha}{n}$. According to the proof of case 1 , we can see that $I_{\alpha}$ is bounded from $M_{s_{1}}^{p_{1}, q_{1}}$ to $M_{s_{2}}^{p_{2}, \tilde{q_{2}}}$. By the condition $q_{2} \geq q_{1}>\tilde{q_{2}}$ and the fact $M_{s_{2}}^{p_{2}, \widetilde{q_{2}}} \hookrightarrow M_{s_{2}}^{p_{2}, q_{2}}$, the proof of Case 2 is completed.

Case 3: $\frac{1}{p_{2}}<\frac{1}{p_{1}}-\frac{\alpha}{n}$. In this case, $0<\frac{1}{p_{1}}-\frac{\alpha}{n}<1$. Take $1<\widetilde{p_{2}}<\infty$ such that $\frac{1}{\widetilde{p_{2}}}=\frac{1}{p_{1}}-\frac{\alpha}{n}$, then $p_{2}>\widetilde{p_{2}}$. Using the embedding result $\widetilde{M_{s_{2}}} \widetilde{p_{2}}, q_{2} \hookrightarrow M_{s_{2}}^{p_{2}}, q_{2}$ and the proof of Case 1 and 2, we finish the proof of Theorem 1.1.

Now, we turn our attention to the proof of Theorem 1.2. From a Fourier point of view, the bilinear fractional integral operator can be rewritten as

$$
\left(\widehat{B_{\alpha}(f, g)}\right)(\xi)=\int_{\mathbb{R}^{n}} g(t) F(t, \xi) e^{-2 \pi i t \xi} d t,
$$

where

$$
F(t, \xi)=\int_{\mathbb{R}^{n}}|y|^{\alpha-n} f(t-2 y) e^{2 \pi i y \xi} d y .
$$

For the proof of Theorem 1.2, we need some lemmas.

Lemma 3.1 Let $H(t, y)=e^{-2 \pi i t \cdot y} \mathcal{F}^{-1} f(y)$ and $\widehat{H_{2}}$ be the Fourier transform for the second variable of $H$, then $\widehat{H_{2}}(t, \xi)=f(t+\xi)$ and $F(t, 2 \xi)=2^{-\alpha} I_{n-\alpha}(H(t, \cdot))(\xi)$.

Proof We only prove the second equality. The first one is very easy, we omit the details here. Note that if we set $y^{\prime}=2 y, F(t, 2 \xi)$ can be rewritten as

$$
\begin{aligned}
F(t, 2 \xi) & =\int_{\mathbb{R}^{n}}\left|\frac{y^{\prime}}{2}\right|^{\alpha-n} f\left(t-y^{\prime}\right) e^{2 \pi i y^{\prime} \xi} 2^{-n} d y^{\prime} \\
& =2^{-\alpha} \int_{\mathbb{R}^{n}}|y|^{\alpha-n} f(t+y) e^{-2 \pi i y \xi} d y=2^{-\alpha}\left(I_{n-\alpha} H(t, \cdot)\right)(-\xi) .
\end{aligned}
$$

We finish the proof of Lemma 3.1. 
Lemma 3.2 Denote $F_{2}^{2}(t, \xi)=F(t, 2 \xi)$, then $\left\|F_{2}^{2}\right\|_{M^{p, q}}=C_{n, p, q}\|F\|_{M^{p, q}}$, where $C_{n, p, q}=$ $2^{-n\left(1+\frac{1}{p}-\frac{1}{q}\right)}$

Proof Taking $g \in \mathcal{S}\left(\mathbb{R}^{2 n}\right) \backslash\{0\}$, then we have

$$
\begin{aligned}
V_{g} F_{2}^{2}(x, \xi, y, \eta) & =\int_{\mathbb{R}^{2 n}} F_{2}^{2}(s, t) \overline{g(s-x, t-\xi)} e^{-2 \pi i(s y+t \eta)} d s d t \\
& =\int_{\mathbb{R}^{2 n}} F(s, 2 t) \overline{g(s-x, t-\xi)} e^{-2 \pi i(s y+t \eta)} d s d t
\end{aligned}
$$

Set $t^{\prime}=2 t$, then

$$
\begin{aligned}
V_{g} F_{2}^{2}(x, \xi, y, \eta) & =2^{-n} \int_{\mathbb{R}^{2 n}} F\left(s, t^{\prime}\right) \overline{\left(s-x, \frac{t^{\prime}-2 \xi}{2}\right)} e^{-2 \pi i\left(s y+\frac{t^{\prime}}{2} \eta\right)} d s d t^{\prime} \\
& =2^{-n} \int_{\mathbb{R}^{2 n}} F\left(s, t^{\prime}\right) \overline{\phi\left(s-x, t^{\prime}-2 \xi\right)} e^{-2 \pi i\left(s y+t^{\prime} \frac{\eta}{2}\right)} d s d t^{\prime} \\
& =2^{-n} V_{\phi} F\left(x, 2 \xi, y, \frac{\eta}{2}\right)
\end{aligned}
$$

where $\phi\left(s, t^{\prime}\right)=g\left(s, \frac{t^{\prime}}{2}\right) \in \mathcal{S}\left(\mathbb{R}^{2 n}\right) \backslash\{0\}$ is another window function. Definition 2.1 gives

$$
\begin{aligned}
\left\|F_{2}^{2}\right\|_{M^{p, q}} & =\left(\int_{\mathbb{R}^{2 n}}\left(\int_{\mathbb{R}^{2 n}}\left|V_{g} F_{2}^{2}(x, \xi, y, \eta)\right|^{p} d x d \xi\right)^{\frac{q}{p}} d y d \eta\right)^{\frac{1}{q}} \\
& =2^{-n}\left(\int_{\mathbb{R}^{2 n}}\left(\int_{\mathbb{R}^{2 n}}\left|V_{\phi} F\left(x, 2 \xi, y, \frac{\eta}{2}\right)\right|^{p} d x d \xi\right)^{\frac{q}{p}} d y d \eta\right)^{\frac{1}{q}}
\end{aligned}
$$

Let $\xi^{\prime}=2 \xi, \eta^{\prime}=\frac{\eta}{2}$. It is easy to check that

$$
\begin{aligned}
\left\|F_{2}^{2}\right\|_{M^{p, q}} & =2^{-n\left(1+\frac{1}{p}-\frac{1}{q}\right)}\left(\int_{\mathbb{R}^{2 n}}\left(\int_{\mathbb{R}^{2 n}}\left|V_{\phi} F(x, \xi, y, \eta)\right|^{p} d x d \xi\right)^{\frac{q}{p}} d y d \eta\right)^{\frac{1}{q}} \\
& =2^{-n\left(1+\frac{1}{p}-\frac{1}{q}\right)}\|F\|_{M^{p, q}}=C_{n, p, q}\|F\|_{M^{p, q}}
\end{aligned}
$$

We complete the proof of Lemma 3.2.

Lemma 3.3 Suppose $\phi_{0}, \phi_{1}, g, h$ to be the non-zero Schwartz functions and $W(g, h)(t, s)=$ $\overline{g(t)} h(s) e^{2 \pi i t s}$, then

$$
V_{W\left(\phi_{0}, \phi_{1}\right)} W(g, h)\left(u_{0}, u, v_{0}, v\right)=e^{-2 \pi i u_{0} u} \overline{V_{\phi_{0}} g\left(u_{0}, u-v_{0}\right)} V_{\phi_{1}} h\left(u, v-u_{0}\right)
$$

Proof The definition of the short time Fourier transform yields

$$
\begin{aligned}
& V_{W\left(\phi_{0}, \phi_{1}\right)} W(g, h)\left(u_{0}, u, v_{0}, v\right) \\
& \quad=\int_{\mathbb{R}^{n}} \int_{\mathbb{R}^{n}} W(g, h)(t, s) \overline{W\left(\phi_{0}, \phi_{1}\right)\left(t-u_{0}, s-u\right)} e^{-2 \pi i\left(t v_{0}+s v\right)} d t d s \\
& \quad=\int_{\mathbb{R}^{n}} \int_{\mathbb{R}^{n}} \overline{g(t)} h(s) e^{2 \pi i t s} \overline{\overline{\phi_{0}\left(t-u_{0}\right)} \phi_{1}(s-u) e^{2 \pi i\left(t-u_{0}\right)(s-u)}} e^{-2 \pi i\left(t v_{0}+s v\right)} d t d s
\end{aligned}
$$




$$
\begin{aligned}
& =e^{-2 \pi i u_{0} u}\left(\int_{\mathbb{R}^{n}} \overline{g(t)} \phi_{0}\left(t-u_{0}\right) e^{2 \pi i\left(u-v_{0}\right) t} d t\right)\left(\int_{\mathbb{R}^{n}} h(s) \overline{\phi_{1}(s-u)} e^{-2 \pi i\left(v-u_{0}\right) s} d s\right) \\
& =e^{-2 \pi i u_{0} u} \overline{V_{\phi_{0}} g\left(u_{0}, u-v_{0}\right)} V_{\phi_{1}} h\left(u, v-u_{0}\right) .
\end{aligned}
$$

The proof of Lemma 3.3 is completed.

Lemma 3.4 For $1<p_{0}<\infty$ and $1 \leq q_{0}, p_{2}, q_{2}<\infty$, let $p_{0}^{\prime}$ and $q_{0}^{\prime}$ denote the conjugate index of $p_{0}$ and $q_{0}$, respectively. If $q_{0}^{\prime} \geq p_{0}^{\prime}$ and $\frac{1}{q_{0}^{\prime}}+\frac{1}{p_{0}^{\prime}}=\frac{1}{p_{2}}+\frac{1}{q_{2}}$, then $W(g, h)$ defined in Lemma 3.3 is bounded from $W\left(\mathcal{F} L^{p_{2}}, L^{p_{0}^{\prime}}\right) \times M^{q_{2}, q_{0}^{\prime}}$ to $M^{p_{0}^{\prime}, q_{0}^{\prime}}$.

Proof For each $g \in W\left(\mathcal{F} L^{p_{2}}, L^{p_{0}^{\prime}}\right)$ and $h \in M^{q_{2}, q_{0}^{\prime}}$, Lemma 3.3 indicates

$$
\begin{aligned}
\|W(g, h)\|_{M^{p_{0}^{\prime}, q_{0}^{\prime}}} \\
\quad=\left(\int_{\mathbb{R}^{2 n}}\left(\int_{\mathbb{R}^{2 n}}\left|V_{W\left(\phi_{0}, \phi_{1}\right)} W(g, h)\left(u_{0}, u, v_{0}, v\right)\right|^{p_{0}^{\prime}} d u_{0} d u\right)^{\frac{q_{0}^{\prime}}{p_{0}^{\prime}}} d v_{0} d v\right)^{\frac{1}{q_{0}^{\prime}}} \\
\quad=\left(\int_{\mathbb{R}^{2 n}}\left(\int_{\mathbb{R}^{2 n}}\left|V_{\phi_{0}} g\left(u_{0}, u-v_{0}\right)\right|^{p_{0}^{\prime}}\left|V_{\phi_{1}} h\left(u, v-u_{0}\right)\right|^{p_{0}^{\prime}} d u_{0} d u\right)^{\frac{q_{0}^{\prime}}{p_{0}^{\prime}}} d v_{0} d v\right)^{\frac{1}{q_{0}^{\prime}}} .
\end{aligned}
$$

Note that $V_{\phi_{0}} g\left(u_{0}, u-v_{0}\right)=e^{-2 \pi i u_{0}\left(u-v_{0}\right)} V_{\widehat{\phi_{0}}} \hat{g}\left(u-v_{0},-u_{0}\right)$. If we denote $P(x, y)=\widetilde{P(-x, y)}$, then

$$
\begin{aligned}
& \|W(g, h)\|_{M^{p_{0}^{\prime}, q_{0}^{\prime}}} \\
& =\left(\int_{\mathbb{R}^{2 n}}\left(\int_{\mathbb{R}^{2 n}}\left|V_{\widehat{\phi_{0}}} \hat{g}\left(u-v_{0},-u_{0}\right)\right|^{p_{0}^{\prime}}\left|V_{\phi_{1}} h\left(u, v-u_{0}\right)\right|^{p_{0}^{\prime}} d u_{0} d u\right)^{\frac{q_{0}^{\prime}}{p_{0}^{\prime}}} d v_{0} d v\right)^{\frac{1}{q_{0}^{\prime}}} \\
& =\left(\int_{\mathbb{R}^{2 n}}\left(\int_{\mathbb{R}^{2 n}}\left|V_{\widehat{\phi_{0}}} \hat{g}\left(\widehat{v_{0}-u},-u_{0}\right)\right|^{p_{0}^{\prime}}\left|V_{\phi_{1}} h\left(u, v-u_{0}\right)\right|^{p_{0}^{\prime}} d u_{0} d u\right)^{\frac{q_{0}^{\prime}}{p_{0}^{\prime}}} d v_{0} d v\right)^{\frac{1}{q_{0}^{\prime}}}
\end{aligned}
$$

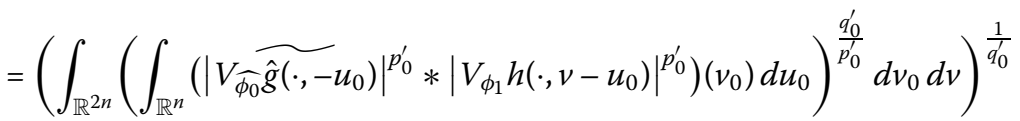

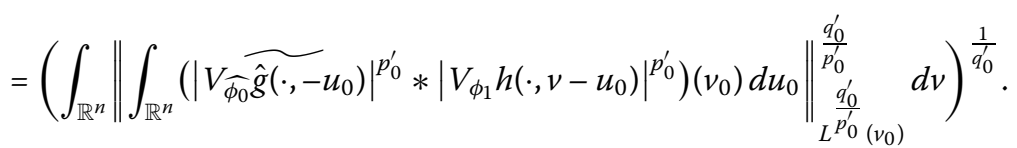

Since $\frac{1}{q_{0}^{\prime}}+\frac{1}{p_{0}^{\prime}}=\frac{1}{p_{2}}+\frac{1}{q_{2}}$, using Minkowski's integral inequalities, Young's inequalities, and Lemma 2.7, we obtain

$$
\begin{aligned}
& \|W(g, h)\|_{M^{p_{0}^{\prime}}, q_{0}^{\prime}}
\end{aligned}
$$

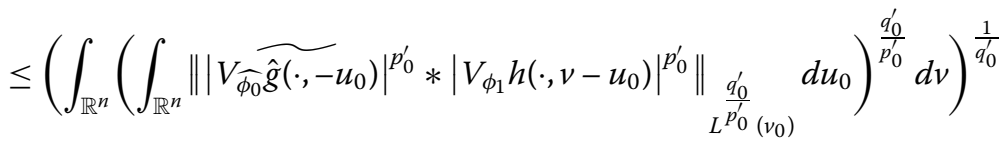

$$
\begin{aligned}
& =\|\hat{g}\|_{M^{p_{2}, p_{0}^{\prime}}}\|h\|_{M^{q_{2}, q_{0}^{\prime}}}=\|g\|_{W\left(\mathcal{F} L^{\left.p_{2}, L^{p_{0}^{\prime}}\right)}\right.}\|h\|_{M^{q_{2}, q_{0}^{\prime}}} .
\end{aligned}
$$

The proof of Lemma 3.4 is completed. 
We give the proof of Theorem 1.2.

Proof Choose $h \in \mathcal{S}\left(\mathbb{R}^{n}\right) \backslash\{0\}$ and denote by $\left.\langle\cdot, \cdot\rangle\right\rangle$ the inner product, then

$$
\begin{aligned}
\left\langle\widehat{B_{\alpha}(f, g)}, h\right\rangle & =\int_{\mathbb{R}^{n}} \widehat{B_{\alpha}(f, g)}(\xi) \overline{h(\xi)} d \xi \\
& =\int_{\mathbb{R}^{n}} \int_{\mathbb{R}^{n}} F(t, \xi) g(t) \overline{h(\xi)} e^{-2 \pi i t \xi} d t d \xi \\
& =\langle F, W(g, h)\rangle,
\end{aligned}
$$

where $W(g, h)=\overline{g(t)} h(\xi) e^{2 \pi i t \xi}$. Hölder's inequalities show

$$
\begin{aligned}
\left|\left\langle\widehat{B_{\alpha}(f, g)}, h\right\rangle\right| & =|\langle F, W(g, h)\rangle| \\
& =\left|\left\langle V_{W\left(\phi_{0}, \phi_{1}\right)} F, V_{W\left(\phi_{0}, \phi_{1}\right)} W(g, h)\right\rangle\right| \\
& \leq\|F\|_{M^{p_{0}, q_{0}}}\|W(g, h)\|_{M^{p_{0}^{\prime}, q_{0}^{\prime}}} .
\end{aligned}
$$

Lemma 3.1, Lemma 3.2, and Theorem 1.1 yield

$$
\begin{aligned}
\|F\|_{M^{p_{0}, q_{0}}} & =C_{n, p_{0}, q_{0}}\left\|F_{2}^{2}\right\|_{M^{p_{0}, q_{0}}}=C_{n, p_{0}, q_{0}}\left\|I_{n-\alpha}(H(t, \cdot))\right\|_{M^{p_{0}, q_{0}}} \\
& \leq C_{n, p_{0}, q_{0}}\|H(t, \cdot)\|_{M^{p_{1}, q_{1}}}=C_{n, p_{0}, q_{0}}\left\|\mathcal{F}^{-1} f\right\|_{M^{p_{1}, q_{1}}} \\
& =C_{n, p_{0}, q_{0}}\|f\|_{W\left(\mathcal{F} L^{p_{1}, L^{\left.q_{1}\right)}} .\right.}
\end{aligned}
$$

On the other hand, Lemma 3.4 gives

$$
\|W(g, h)\|_{M^{p_{0}^{\prime}, q_{0}^{\prime}}} \leq C\|g\|_{W\left(\mathcal{F} L^{\left.p_{2}, L^{p_{0}^{\prime}}\right)}\right.}\|h\|_{M^{q_{2}, q_{0}^{\prime}}} \cdot
$$

Therefore,

$$
\left|\left\langle\widehat{B_{\alpha}(f, g)}, h\right\rangle\right| \leq C_{n, p_{0}, q_{0}}\|f\|_{W\left(\mathcal{F} L^{\left.p_{1}, L^{q_{1}}\right)}\right.}\|g\|_{W\left(\mathcal{F} L^{\left.p_{2}, L^{p_{0}^{\prime}}\right)}\right.}\|h\|_{M^{q_{2}, q_{0}^{\prime}}},
$$

which implies $\widehat{B_{\alpha}(f, g)} \in M^{q_{2}^{\prime}, q_{0}}$ and

$$
\left\|\widehat{B_{\alpha}(f, g)}\right\|_{M^{q_{2}^{\prime}, q_{0}}} \leq C_{n, p_{0}, q_{0}}\|f\|_{W\left(\mathcal{F} L^{\left.p_{1}, L^{q_{1}}\right)}\right.}\|g\|_{W\left(\mathcal{F} L^{\left.p_{2}, L^{p_{0}^{\prime}}\right)}\right.} .
$$

Lemma 2.7 indicates $\left\|B_{\alpha}(f, g)\right\|_{W\left(\mathcal{F} L^{q_{2}^{\prime}, L} q_{0}\right)}=\left\|\widehat{B_{\alpha}(f, g)}\right\|_{M^{q_{2}^{\prime}, q_{0}}}$, we conclude

$$
\left\|B_{\alpha}(f, g)\right\|_{W\left(\mathcal{F} L^{q_{2}, L^{\left.q_{0}\right)}}\right.} \leq C_{n, p_{0}, q_{0}}\|f\|_{W\left(\mathcal{F} L^{\left.p_{1}, L^{q_{1}}\right)}\right.}\|g\|_{W\left(\mathcal{F} L^{p_{2}, L^{p_{0}^{\prime}}}\right.} .
$$

The proof of Theorem 1.2 is finished.

The authors declare that they have no competing interests. 


\section{Acknowledgements}

This work is supported by the National Nature Science Foundation of China (No. 11201003) and NNSF (No. KJ2014A087) of Anhui Province in China.

Received: 8 July 2015 Accepted: 16 October 2015 Published online: 29 October 2015

\section{References}

1. Feichtinger, HG, Gröchenig, K: Gabor frames and time-frequency analysis of distributions. J. Funct. Anal. 146, 464-495 (1997)

2. Gröchenig, K, Heil, C, Okoudjou, K: Gabor analysis in weighted amalgam spaces. Sampl. Theory Signal Image Process., Int. J. 1, 225-259 (2002)

3. Feichtinger, HG: Banach spaces of distributions of Wiener's type and interpolation. In: Butzer, P, Nagy, BS, Görlich, E (eds.) Proc. Conf. Oberwolfach, Functional Analysis and Approximation, August 1980. Ins. Ser. Num. Math., vol. 69, pp. 153-165. Birkhäuser, Basel (1981)

4. Feichtinger, HG: Convolution algebra of Wiener's type. In: Proc. Conf. Functions, Series, Operators, Budapest, August 1980. Colloq. Math. Soc. János Bolyai, vol. 35, pp. 509-524. North-Holland, Amsterdam (1983)

5. Feichtinger, HG: Modulation spaces on locally compact Abelian groups. Technical Report. University of Vienna, Vienna (1983)

6. Fournier, JJF, Stewart, J: Amalgams of $L^{p}$ and $1^{9}$. Bull. Am. Math. Soc. (N.S.) 13, 1-21 (1985)

7. Heil, C: An introduction to weighted Wiener amalgams. In: Krishna, M, Radha, R, Thangavelu, S (eds.) Wavelets and Their Applications, pp. 183-216. Allied Publishers Private Limited, New Delhi (2003)

8. Bényi, A, Okoudjou, KA: Modulation space estimates for multilinear pseudodifferential operators. Stud. Math. 172 169-180 (2006)

9. Bényi, A, Okoudjou, KA: Local well-posedness of nonlinear dispersive equations on modulation spaces. Bull. Lond. Math. Soc. 41, 549-558 (2009)

10. Cordero, E, Nicola, F: Sharpness of some properties of Wiener amalgam and modulation spaces. Bull. Aust. Math. Soc. 80, 105-116 (2009)

11. Cordero, E, Nicola, F: Metaplectic representation on Wiener amalgam spaces and applications to Schrödinger equation. J. Funct. Anal. 254, 506-534 (2008)

12. Cordero, E, Nicola, F: Some new Strichartz estimates for the Schrödinger equation. J. Differ. Equ. 245, 1945-1974 (2008)

13. Cordero, E, Nicola, F: Strichartz estimates in Wiener amalgam spaces for the Schrödinger equations. Math. Nachr. 28 25-41 (2008)

14. Cordero, E, Nicola, F: Pseudodifferential operators on $L^{p}$, Wiener amalgam and modulation spaces. Int. Math. Res. Not. 10, 1860-1893 (2010)

15. Feichtinger, HG, Narimani, G: Fourier multipliers of classical modulation spaces. Appl. Comput. Harmon. Anal. 21, 349-359 (2006)

16. Kobayashi, M: Multipliers on modulation spaces. SUT J. Math. 42, 305-312 (2006)

17. Okoudjou, K: Embeddings of some classical Banach spaces into the modulation spaces. Proc. Am. Math. Soc. 132, 1639-1647 (2004)

18. Toft, J: Continuity properties for modulation spaces with applications to pseudo-differential calculus. I. J. Funct. Anal. 207, 399-429 (2004)

19. Wang, B, Hudzik, H: The global Cauchy problem for the NLS and NLKG with small rough data. J. Differ. Equ. 232, 36-73 (2007)

20. Tomia, N: Fractional integrals on modulation spaces. Math. Nachr. 279, 672-680 (2006)

21. Sugimoto, M, Tomia, N: A remark on fractional integrals on modulation spaces. Math. Nachr. 281, 1372-1379 (2008)

22. Chen, J, Zhong, Y: Modulation space estimates for the fractional integral operators. Sci. China Math. 54, 1478-1489 (2011)

23. Kobayashi, M: Modulation spaces $M^{p, q}$ for $0<p, q \leq \infty$. J. Funct. Spaces Appl. 4, 329-341 (2006)

24. Gröchenig, K, Foundations of Time-Frequency Analysis. Birkhäuser, Boston (2001)

25. Kobayashi, M: Dual of modulation spaces. J. Funct. Spaces Appl. 5, 1-8 (2007)

\section{Submit your manuscript to a SpringerOpen ${ }^{\circ}$ journal and benefit from:}

- Convenient online submission

Rigorous peer review

- Immediate publication on acceptance

- Open access: articles freely available online

- High visibility within the field

- Retaining the copyright to your article 